Journal of Universal Mathematics

Vol.4 No.2 PP.222-229 (2021)

ISSN-2618-5660

DOI: $10.33773 /$ jum. 952394

\title{
C-PURE SUBMODULES AND C-FLAT MODULES
}

\author{
YUSUF ALAGÖZ \\ 0000-0002-2535-4679
}

\begin{abstract}
Let $R$ be a ring. A right $R$-module $A$ is said to be $\mathrm{C}$-flat if the kernel of any epimorphism $B \rightarrow A$ is C-pure in $B$, i.e. the induced map $\operatorname{Hom}(C, B) \rightarrow \operatorname{Hom}(C, A)$ is surjective for any cyclic right $R$-module $C$. Projective modules are C-flat and $\mathrm{C}$-flat modules are weakly-flat and neat-flat. In this article, it is discussed the connections between C-flat, weakly-flat, neatflat and singly flat modules. It is shown that C-flat modules coincide with singly-projective modules over arbitrary rings. Next, several characterizations of certain classes of rings and modules via C-purity are considered. We prove that every C-flat module is injective if and only if $R$ is a QF ring. Moreover, we show that $R$ is a CF ring if and only if every FP-injective right $R$-module is C-flat.
\end{abstract}

\section{INTRODUCTION}

Throughout, $R$ will denote an associative ring with identity, and modules will be unital $R$-modules unless otherwise stated.

There are many submodule structures, but the most commonly studied structures are closed submodule and pure submodule, due to their important role played in Module and Ring Theory. A submodule $B$ of a right $R$-module $A$ is called closed (in $A$ ) provided $B$ has no proper essential extension in $A$. Let $\varepsilon: 0 \rightarrow B \rightarrow A \rightarrow$ $C \rightarrow 0$ be an exact sequence of right $R$-modules. $\varepsilon$ is called (Cohn) pure exact if, every finitely presented right $R$-module $F$ is projective with respect to $\varepsilon$ (see [22]). The sequence $\varepsilon$ is called C-pure (resp. neat) if every cyclic (resp. simple) right $R$-module is projective with respect to $\varepsilon$ (see $[19,16]$, respectively). C-pure (resp. neat) and pure are in general inequivalent, none implies the other. In general, Cpure submodules are closed and closed submodules are neat, and the converses are true if $\mathrm{R}$ is a right CPS ring, i.e. every cyclic right $R$-module is a direct sum of a projective module and a semisimple module (see [9]).

Recently, there is a significant interest in some classes of modules that are defined via closed submodules, neat submodules and C-pure submodules (see [1, 4, 6, 23]). A right R-module $A$ is called weakly-flat [23](resp. neat-flat [4]) if the kernel of any epimorphism $B \rightarrow A$ is closed (resp. neat) in $B$.

Date: Received: 2021-06-14; Accepted: 2021-07-29.

2000 Mathematics Subject Classification. 16D40, 16D50, 16E10.

Key words and phrases. Singly projective modules, C-pure submodules, CPS rings. 
In this article, motivated by the weakly-flat and neat-flat modules, we continue the study and investigation of modules $A$, for which any short exact sequence ending with $A$ is C-pure. Namely, a right $R$-module $A$ is said to be C-flat if the kernel of any epimorphism $B \rightarrow A$ is C-pure in $B$, i.e. the induced map $\operatorname{Hom}(C, B) \rightarrow \operatorname{Hom}(C, A)$ is surjective for any cyclic right $R$-module $C$ ([11]). Projective modules are C-flat and C-flat modules are weakly-flat and neat-flat. It is discussed the connections between C-flat, weakly-flat and neat-flat modules. In [3], a right $R$-module $A$ is called singly-projective if for any cyclic right $R$-module $C$, every homomorphism $f: C \rightarrow A$ factors through a finitely generated free right $R$-module $F$. It is shown that $\mathrm{C}$-flat modules coincide with singly-projective modules over arbitrary rings. Next, several characterizations of certain classes of rings and modules via C-purity are considered. We prove that every $\mathrm{C}$-flat module is injective if and only if $R$ is a $\mathrm{QF}$ ring. Moreover, we show that $R$ is a $\mathrm{CF}$ ring if and only if every FP-injective right $R$-module is C-flat.

\section{C-Flat modules}

Let $\varepsilon: 0 \rightarrow B \stackrel{f}{\longrightarrow} A \stackrel{g}{\longrightarrow} C \rightarrow 0$ be an exact sequence of right $R$-modules. $\varepsilon$ is called $C$-pure exact if, $f(B)$ is a C-pure submodule of $A$ (see [19]). In this case, $f$ and $g$ are called C-pure monomorphism and C-pure epimorphism, respectively. By definition, the class of C-pure exact sequences is projectively generated by the class of cyclic right $R$-modules. Hence C-pure exact sequences form a proper class in the sense of Bushbaum, (see [11, Proposition 1.7]).

Proposition 1. The following are equivalent for a right $R$-module $A$.

(1) $A$ is C-flat.

(2) Every exact sequence $0 \rightarrow C \rightarrow B \rightarrow A \rightarrow 0$ is C-pure.

(3) There exists a C-pure exact sequence $0 \rightarrow C \rightarrow P \rightarrow A \rightarrow 0$ with $P$ projective.

(4) There exists a C-pure exact sequence $0 \rightarrow C \rightarrow F \rightarrow A \rightarrow 0$ with $F$ C-flat.

Proof. (1) $\Rightarrow(2) \Rightarrow(3) \Rightarrow(4)$ are clear.

(4) $\Rightarrow$ (1) Let $0 \rightarrow C \rightarrow B \stackrel{g}{\longrightarrow} A \rightarrow 0$ be any short exact sequence. We claim that $g$ is a C-pure epimorphism, i.e., $\operatorname{Ker}(g)$ is a C-pure submodule of $B$. By (4), there exists a C-pure exact sequence $0 \rightarrow C \rightarrow F \stackrel{h}{\longrightarrow} A \rightarrow 0$ with $F$ C-flat. Considering the pullback of $g$ and $h$, we obtain a commutative diagram with exact rows

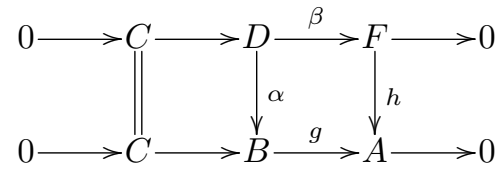

Since $F$ is C-flat, $\beta$ is a C-pure epimorphism. Also, since $h$ is C-pure epimorphism, $g \alpha=h \beta$ is again C-pure epimorphism. This means that $g$ is a C-pure epimorphism by [11, Proposition 1.7], and this completes the proof.

Remark 2.1. (1) If an R-module $A$ is C-flat then $A$ is torsion-free by [11, Proposition 4.3 but not conversely. If $R$ is a commutative integral domain, then every torsionfree module is C-flat by [11, Proposition 4.4]. 
(2) Obviously, projective modules are C-flat. But the converse is not true in general. Let $R=k[X, Y]$ be a polynomial ring in two variables $X, Y$ over a field $k$. Here the ideal $(X, Y)$ of $R$ is torsion-free, and so is C-flat by (1) since $R$ is a commutative integral domain. But it is not flat as $R$-module by [2, Chapter I, Exercise 2.3], and so is not projective.

(3) C-flat and flat are in general inequivalent and none implies the other by [11, Remarks 4.6 and 4.7].

(4) Note that a cyclic right $R$-module is C-flat if and only if it is projective. Thus $R$ is a semisimple Artinian ring if and only if every right $R$-module is C-flat.

The following observation is easy to show by Proposition 1 and useful for the further characterization of C-flat modules.

Lemma 2.2. Let $R$ be a ring. An $R$-module $A$ is singly projective if and only if $A$ is $C$-flat.

Let $A$ be a finitely presented right $R$-module. Then there exists a short exact sequence $0 \rightarrow G \rightarrow F \rightarrow A \rightarrow 0$ with $F$ and $G$ finitely generated free. If we apply the functor $\operatorname{Hom}_{R}(-, R)$ to this exact sequence, we obtain the sequence $0 \rightarrow A^{*} \rightarrow F^{*} \rightarrow G^{*} \rightarrow \operatorname{Tr}(A) \rightarrow 0$ where $\operatorname{Tr}(A)$ is the cokernel of the dual map $F^{*} \rightarrow G^{*}$. Note that $\operatorname{Tr}(A)$ is a finitely presented left $R$-module. The left $R$-module $\operatorname{Tr}(A)$ is called an Auslander-Bridger Transpose of the right $R$-module $A$ (see $[20, \S 5]$ ). Over a right Noetherian ring, every cyclic right $R$-module $C$ and its transpose $\operatorname{Tr}(C)$ are finitely presented.

Proposition 2. Let $R$ be a right Noetherian ring and $A$ a right $R$-module. Then $A$ is C-flat if and only if $\operatorname{Tor}_{1}(A, \operatorname{Tr}(C))=0$ for each cyclic right $R$-module $C$.

Proof. Let $0 \rightarrow G \rightarrow F \rightarrow A \rightarrow 0$ be an exact sequence with $F$ projective. If we assume that $A$ is C-flat right $R$-module, then $0 \rightarrow \operatorname{Hom}(C, G) \rightarrow \operatorname{Hom}(C, F) \rightarrow$ $\operatorname{Hom}(C, A) \rightarrow 0$ is exact for any cyclic right $R$-module $C$. Since $R$ is right Noetherian, $C$ is finitely presented, and so $0 \rightarrow G \otimes \operatorname{Tr}(C) \rightarrow F \otimes \operatorname{Tr}(C)$ is left exact by [20, Theorem 8.3]. Hence $\operatorname{Tor}_{1}^{R}(A, \operatorname{Tr}(C))=0$. Conversely, suppose $\operatorname{Tor}_{1}^{R}(A, \operatorname{Tr}(C))=$ 0 for each cyclic right $R$-module $C$. Thus $0 \rightarrow G \otimes \operatorname{Tr}(C) \rightarrow F \otimes \operatorname{Tr}(C)$ is left exact, and so $0 \rightarrow \operatorname{Hom}(C, G) \rightarrow \operatorname{Hom}(C, F) \rightarrow \operatorname{Hom}(C, A) \rightarrow 0$ is exact again by [20, Theorem 8.3]. This means that $A$ is C-flat by Proposition 1.

Recall that a right $R$-module $A$ is called singly injective if $\operatorname{Ext}_{R}^{1}(F / K, A)=0$ for any cyclic submodule $K$ of any finitely generated free right $R$-module $F$. A right $R$-module $A$ is called singly flat if $\operatorname{Tor}_{R}^{1}(A, F / K)=0$ for any cyclic submodule $K$ of any finitely generated free right $R$-module $F$ (see [14]).

Proposition 3. Let $R$ be a right Noetherian ring and $A$ a left $R$-module. $A$ is singly injective if and only if $\operatorname{Ext}_{R}^{1}(\operatorname{Tr}(C), A)=0$ for any cyclic right $R$-module $C$.

Proof. Let $0 \rightarrow A \rightarrow E(A) \rightarrow E(A) / A \rightarrow 0$ be an exact sequence. If we assume that $A$ is singly injective left $R$-module, then $0 \rightarrow C \otimes A \rightarrow C \otimes E(A) \rightarrow C \otimes E(A) / A \rightarrow$ 0 is exact by [7, Lemma 2.1 and Proposition 2.2]. So $\operatorname{Hom}(\operatorname{Tr}(C), E(A)) \rightarrow$ $\operatorname{Hom}(\operatorname{Tr}(C), E(A) / A)$ is epic by [20, Theorem 8.3], whence $\operatorname{Ext}_{R}^{1}(\operatorname{Tr}(C), A)=0$. Conversely, if we assume that $\operatorname{Ext}_{R}^{1}(\operatorname{Tr}(C), A)=0$ for each cyclic right $R$-module $C$, then $\operatorname{Hom}(\operatorname{Tr}(C), E(A)) \rightarrow \operatorname{Hom}(\operatorname{Tr}(C), E(A) / A)$ is epic. So $0 \rightarrow C \otimes A \rightarrow$ $C \otimes E(A)$ is left exact by [20, Theorem 8.3], whence $A$ is singly injective by $[7$, Lemma 2.1 and Corollary 2.6]. 
Corollary 1. Let $R$ be a right Noetherian ring. Then the following are true:

(1) $A$ is C-flat right $R$-module if and only if $A^{+}$is singly injective.

(2) $A$ is singly-injective left $R$-module if and only if $A^{+}$is C-flat.

(3) $A$ is C-flat right $R$-module if and only if $A$ is singly flat.

(4) $A$ is C-flat right $R$-module if and only if $A^{++}$is C-flat.

Proof. (1) For any cyclic right $R$-module $C, \operatorname{Tr}(C)$ is finitely presented. Thus the result follows by the standard isomorphism $\operatorname{Ext}_{R}^{1}\left(\operatorname{Tr}(C), A^{+}\right) \cong \operatorname{Tor}_{1}(A, \operatorname{Tr}(C))^{+}$ and Propositions 2 and 3.

(2) Let $A$ be a left $R$-module and $C$ a cyclic right $R$-module. Then we have $\operatorname{Tor}_{1}\left(A^{+}, \operatorname{Tr}(C)\right) \cong \operatorname{Ext}_{R}^{1}(\operatorname{Tr}(C), A)^{+}$by [18, Theorem 9.51]. Hence the result follows also by Propositions 2 and 3 .

(3) follows by (1) and [14, Lemma 2.4].

(4) follows by (1) and (2).

Recall that a ring $R$ is said to be left hereditary (respectively, left semihereditary, left PP) if every left ideal (respectively, finitely generated left ideal, principal left ideal) of $R$ is projective. A right $R$-module $A$ is called FP-injective if $\operatorname{Ext}_{R}^{1}(F / K, A)=0$ for any finitely generated submodule $K$ of any finitely generated free right $R$-module $F$ (see [13]).

Corollary 2. The following are equivalent for a right Noetherian ring $R$.

(1) Every singly injective left $R$-module is FP-injective.

(2) Every C-flat right $R$-module is flat.

Moreover, if $R$ is a commutative $\mathrm{PP}$ ring, then the above conditions are equivalent to:

(3) $R$ is hereditary.

Proof. (1) $\Leftrightarrow(2)$ follows by Corollary 1 and [14, Corollary 2.11].

(1) $\Leftrightarrow$ (3) follows by [7, Theorem 3.9] and by the fact that Noetherian semihereditary rings are hereditary.

In [12], a ring $R$ is called right CPS if every cyclic right $R$-module is a direct sum of a projective module and a semisimple module.

Remark 2.3. (1) Following [9], C-pure submodules are closed, but not conversely.

(2) Since closed submodules are neat by [21], C-pure submodules are neat.

(3) A ring $R$ is right CPS ring if and only if neat submodules are C-pure. In particular, if $R$ is a right CPS ring, then closed submodules are also C-pure (see $[9])$.

Since C-pure submodules are closed and closed submodules are neat, we have the following implications in our concepts:

C-flat $\Rightarrow$ weakly flat $\Rightarrow$ neat-flat.

Recall that a ring $R$ is said to be a right $C$-ring if $\operatorname{Soc}(R / I) \neq 0$ for every essential right ideal $I$ of $R$. Right CPS rings, left perfect rings and right semiartinian rings are well known examples of right $C$-rings $([5,10.10])$. Together with Remark 2.3(3) and $[4$, Proposition 2.9], we obtain the following.

Corollary 3. Let $R$ be a right CPS ring and $A$ be a right $R$-module. The following statements are equivalent:

(1) $A$ is C-flat. 
(2) $A$ is weakly-flat.

(3) $A$ is neat-flat.

(4) $\operatorname{Soc}(A)=A \cdot \operatorname{Soc}\left(R_{R}\right)$.

A right $R$-module $M$ is called $C S$ if every closed submodule of $M$ is a direct summand of $M$ and a ring $R$ called right $C S$ if $R_{R}$ is $C S$. A ring $R$ is called right $\Sigma-C S$ (respectively, right finitely $\Sigma-C S$ ) if every (respectively, finite) direct sum of copies of $R_{R}$ is $C S$ (the reader might consult [8]).

Proposition 4. If every neat-flat right $R$-modules is C-flat, then $R$ is a right CS and right $C$-ring.

Proof. The hypothesis implies that every neat-flat right $R$-module weakly-flat, and so $R$ is a $C$-ring by [4, Proposition 2.7]. Let $I$ be a closed right ideal of $R$. Then $I$ is neat in $R$, and so $R / I$ is neat-flat by [4, Lemma 2.1]. Thus by the hypothesis, $R / I$ is C-flat and also $0 \rightarrow I \rightarrow R \rightarrow R / I \rightarrow 0$ is C-pure. Since $R / I$ is projective with respect to $\mathrm{C}$-pure exact sequences, the exact sequence $0 \rightarrow I \rightarrow R \rightarrow R / I \rightarrow 0$ splits. Thus $I$ is a direct summand of $R$, that is $R$ is a right $C S$ ring.

Recall by [17] that a ring $R$ is a right SC-ring if every cyclic singular right $R$ module is semisimple. By Remark 2.3(3) and [4, Proposition 2.9], we obtain the following.

Corollary 4 . Let $R$ be a right SC-ring. The following are equivalent:

(1) $R$ is right CPS.

(2) Every neat-flat right $R$-module is C-flat.

(3) Every weakly-flat right $R$-module is C-flat.

Proof. $(1) \Rightarrow(2)$ This follows directly by Corollary $3 .(2) \Rightarrow(3)$ is clear.

$(3) \Rightarrow(1)$ Let $I$ be a closed right ideal of $R$. Then $R / I$ is weakly-flat. Thus by the hypothesis, $R / I$ is $\mathrm{C}$-flat and also $0 \rightarrow I \rightarrow R \rightarrow R / I \rightarrow 0$ is C-pure. Similar to the proof of Proposition $4, R$ is a right $C S$ ring. Thus $R$ is a right CPS ring by the fact that $R$ is a right SC-ring (see [12, Corollary 4.4]).

Remark 2.4. If $R$ is a right $\Sigma-C S$ ring, then every C-flat right $R$-module is projective by [4, Theorem 2.10.]. The converse is also true if $R$ is a right CPS-ring by Corollary 3 and [4, Theorem 2.10].

Proposition 5. If $R$ is right finitely $\Sigma$-CS, then every finitely generated C-flat right $R$-module is projective.

Proof. Let $A$ be a finitely generated C-flat right $R$-module and consider the short exact sequence $\varepsilon: 0 \rightarrow K \rightarrow F \rightarrow A \rightarrow 0$ with $F$ finitely generated free. By Proposition $1, \varepsilon$ is $\mathrm{C}$-pure, and so is closed. This means that $\varepsilon$ splits by the hypothesis. Thus $A$ is projective.

A ring $R$ is called right $\mathrm{CF}$ if every cyclic right $R$-module embeds in a free module. $R$ is said to be a left AFG ring in case the left annihilator of every nonempty subset of $R$ is a finitely generated left ideal, equivalently every right $R$-module has a singly projective preenvelope (see [15]).

Proposition 6. Let $R$ be a ring. The following are equivalent:

(1) $R$ is right $\mathrm{CF}$.

(2) Every FP-injective right $R$-module is C-flat. 
(3) Every injective right $R$-module is C-flat.

Moreover, if $R$ is left $\mathrm{AFG}$, then the above conditions are equivalent to:

(4) Every right $R$-module has a monic C-flat preenvelope.

Proof. (1) $\Rightarrow(2)$ Let $E$ be an FP-injective right $R$-module and $C$ a cyclic right $R$-module. Since $R$ is a CF ring, $C$ can be embedded in a finitely generated free right $R$-module $F$. Consider the inclusion map $i: C \hookrightarrow F$ and a homomorphism $f: C \rightarrow E$. As $E$ is FP-injective, there exists a homomorphism $g: F \rightarrow E$ such that $g i=f$. Thus $E$ is C-flat by Lemma 2.2 and [15, Lemma 2.1].

$(2) \Rightarrow(3)$ Since injective modules are FP-injective, it is clear.

$(3) \Leftrightarrow(4) \Rightarrow(1)$ Follows by Lemma 2.2 and [15, Lemma 3.6].

Recall that $R$ is said to be a $Q F$-ring if $R$ is left Noetherian and left self-injective, or equivalently every injective (resp. projective) right $R$-module is projective (resp. injective) (see [10]). In the following result, we give a new characterization of a $Q F$ ring.

Proposition 7. Let $R$ be a ring. The following are equivalent:

(1) $R$ is a $Q F$ ring.

(2) $R$ is a right $\mathrm{CF}$ ring and every $\mathrm{C}$-flat right $R$-module is projective.

(3) Every C-flat right $R$-module is injective.

Proof. (1) $\Rightarrow(2)$ It is clear that $R$ is a right $\mathrm{CF}$ and right $\Sigma$-CS ring. Thus (2) follows by Remark 2.4.

$(2) \Rightarrow(1)$ is clear by Proposition 6 .

$(1) \Rightarrow(3)$ Let $A$ be a C-flat right $R$-module. Since $R$ is $Q F, R$ is right $\Sigma-C S$, and so $A$ is projective by Remark 2.4. Being $R$ is $Q F$ implies that $A$ is injective.

$(3) \Rightarrow(1)$ Clear since projective right modules are C-flat.

\section{Conclusion}

In this paper, we continue the study and investigation of C-flat modules and we discuss the connections between C-flat, weakly-flat, neat-flat and singly flat modules. Then we investigate basic properties of the C-flat modules and some characterizations of $\mathrm{CF}$ and $\mathrm{QF}$ rings. We show that C-flat modules coincide with singly-projective modules over arbitrary rings. This work provides a new approach to singly projective modules in terms of C-pure submodules.

\section{Acknowledgments}

The authors would like to thank the reviewers and editors of Journal of Universal Mathematics.

\section{Funding}

The author(s) declared that has no received any financial support for the research, authorship or publication of this study.

The Declaration of Conflict of Interest/ Common Interest

The author(s) declared that no conflict of interest or common interest

The Declaration of Ethics Committee Approval 
This study does not be necessary ethical committee permission or any special permission.

\section{The Declaration of Research and Publication Ethics}

The author(s) declared that they comply with the scientific, ethical, and citation rules of Journal of Universal Mathematics in all processes of the study and that they do not make any falsification on the data collected. Besides, the author(s) declared that Journal of Universal Mathematics and its editorial board have no responsibility for any ethical violations that may be encountered and this study has

not been evaluated in any academic publication environment other than Journal of Universal Mathematics.

\section{REFERENCES}

[1] Y. Alagöz, E. Büyükaşık, On max-flat and max-cotorsion modules. AAECC, 32, 195-215, (2021).

[2] F. W. Anderson, K. R. Fuller, Rings and Categories of Modules. 2nd ed. Grad. Texts in Math., Vol. 13. Berlin: Springer-Verlag. (1992).

[3] G. Azumaya, Finite splitness and finite projectivity. J. Algebra, 106, 114-134, (1987).

[4] E. Büyükaşık, Y. Durğun, Neat-flat modules. Comm. Algebra, 44(1), 416-428, (2016).

[5] J. Clark, C. Lomp, N. Vanaja and R. Wisbauer, Lifting modules. Frontiers in Mathematics, Birkhäuser Verlag, Basel, (2006).

[6] S. Crivei, Neat and coneat submodules of modules over commutative rings. Bull. Aust. Math. Soc. 89(2), 343-352, (2014).

[7] A. Moradzadeh-Dehkordi and S. H. Shojaee, Singly injective modules, J. Algebra Appl. 1820(1), 1950007, (2019).

[8] N. V. Dung, D.V. Huynh, P. F. Smith and R. Wisbauer, Extending modules, Putman Research Notes in Mathematics Series, Longman, Harlow, (1994).

[9] Y. Durğun, Projectivity relative to closed (neat) submodules. J. Algebra Appl., DOI: 10.1142/S0219498822501146, (2021).

[10] C. Faith, Algebra. II, Springer-Verlag, Berlin-New York. Ring theory, Grundlehren der Mathematischen Wissenschaften, No. 191, (1976).

[11] V. A. Hiremath, S. S. Gramopadhye, Cyclic Pure Submodules. Int. J. Alg., 3(3), 125-135, (2009).

[12] D. V. Huynh, and P. Dân, On rings with restricted minimum condition. Arch. Math.(Basel), 51, 313-326, (1988).

[13] B.H. Maddox, Absolutely pure modules, Proc. Amer. Math. Soc. 18, 155-158, (1967).

[14] L. Mao and N. Ding, New characterizations of pseudo-coherent rings, Forum Math. 22, 993-1008, (2010).

[15] L. Mao, A generalization of noetherian rings. Taiwanese J. Math., 12(2), 501-512, (2008).

[16] G. Renault, Étude de certains anneaux a liés aux sous-modules compléments dun a-module,

C. R. Acad. Sci. Paris, 259, 4203-4205, (1964).

[17] S. T. Rizvi and M. F. Yousif, On continuous and singular modules, Noncommutative ring theory (Athens, OH, 1989), pp. 116-124,(1990).

[18] J. J. Rotman, An Introduction to Homological Algebra, in Pure Appl.Math., Vol. 85, Academic Press, New York, (1979).

[19] G. Simmons, Cyclic-purity: a generalization of purity for modules. Houston J. Math., 13(1), 135-150, (1987).

[20] E.G. Sklyarenko, Relative Homological Algebra in Categories of Modules. Russian Math. Surveys 33(3):97-137. Translated from Russian from Uspehi Mat. Nauk 33(201):85-120, (1978).

[21] B. Stenström, High submodules and purity. Arkiv för Matematik, 7(11), 173-176, (1967).

[22] R.B. Warfield Jr., Purity and algebraic compactness for modules, Pacific J. Math. 28, 699-719, (1969).

[23] H. Zöschinger, Schwach-Flache moduln. Comm. Algebra, 41(12), 4393-4407, (2013). 
(Yusuf Alagöz) Sirrt University, Department of Mathematics, Simrt, Turkey

Email address: yusuf.alagoz@siirt.edu.tr 